\title{
Research of Nordic Furniture Design
}

\author{
Fangli Li \\ Lanzhou Jiaotong University, Lanzhou, Gansu, 730000
}

Keywords: Nordic Furniture, Features, Origin

\begin{abstract}
From the 1930s to the 1950s, Nordic furniture design was legendary development, the unique Nordic "functionalism" concept is also perfect during this period, and the emergence of a number of furniture designers, driven by these masters, Nordic countries of the furniture design gradually formed a relatively uniform style and swept the world. Today, Scandinavian designers and manufacturers are no longer focused solely on the appearance of furniture, but rather focus on presenting the designer's unique style. For a long time, the "Nordic Design", which is known as "concise, comfortable and modern", is indeed a style that can not be overlooked in the world design field. To understand this style in a comprehensive way, Style behind the reasons, because a regional design style is produced by the region's natural environment and social factors and other conditions.
\end{abstract}

\section{Introduction}

People living and working space and the room is a broad sense of "indoors", it is an important part of human society, a room scale, proportion, light, color composition and so on affect the style and quality of space. Furniture as the main body of the indoor furnishings system, furniture plays an important role, it is the most intimate friend of human life, always affecting people's thinking and behavior.

Nordic is located in the edge of Europe, the culture is also natural edge, furniture design by the European Baroque, Rococo design ideas are not too much, so can always maintain a simple design principles. This determines that the Nordic design tradition itself contains concise and clear, functional first principles. In Scandinavia, furniture design is not in the traditional sense of the scope of industrial design or product design, but is understood as part of the space, and always affect the indoor atmosphere. Here, the meaning of interior design is the first furniture design, they believe that furniture is for people's lives and work services, and therefore must be consistent with the human scale, ideas and psychology. Furniture and the indoor space relationship may be a unique Nordic phenomenon, but also the Nordic furniture design simple and practical roots.

\section{History of Nordic furniture design}

The so-called Nordic geographically refers to the five countries in the cold northern regions of Sweden, Denmark, Finland, Norway and Iceland, often referred to as the Scandinavian region. (Figure 1) Scandals and climatic conditions in the Nordic region are extremely severe, geographically close to the Arctic, the cold weather, the long winter so that the Nordic people stay at home more, they pay great attention to home life, make the family more comfortable, One of the national traditions of each country. The secluded state of the Nordic region has given the Gospel of the Scandinavian people and brought them inconvenience.

Scandinavians combine their love and worship with nature with their own frugality and simplicity of love, creating a unique style of home decoration. The color combinations they use are comprehended from the changes in natural chromaticity. White on the background dotted with bright wood, and with yellow, green, red and blue to be emphasized. Use flowers as the basic pattern of patterns, or with simple box, stripes and checkerboard grid pattern. (Figure 2) They mix the lines of new furniture and more gorgeous ancestral furniture mixed together. (Figure 3) In accordance with the "less is more" philosophy, the Nordic craftsmen to the various historical period 
of furniture arranged a sense of harmony. In fact, through this arrangement, each furniture can show their own style.

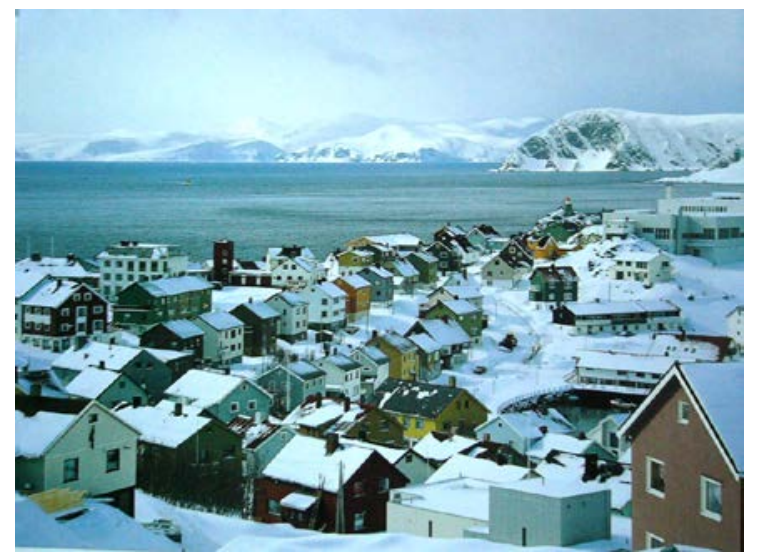

Figure 1 Scandinavian scenery

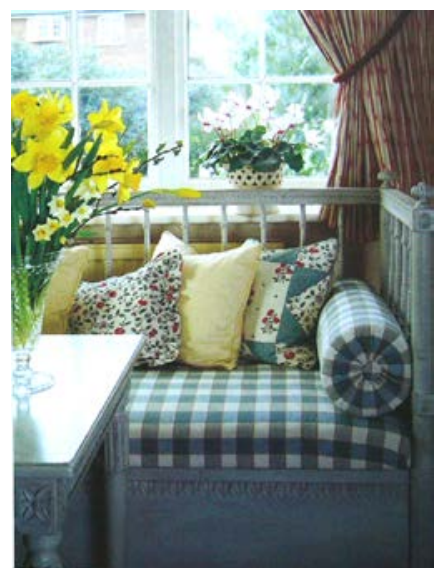

Figure 2 Nordic interior layout

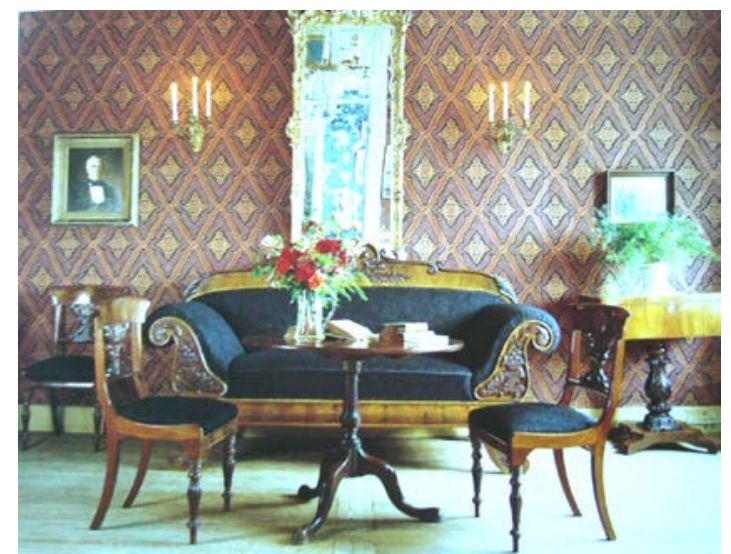

Figure 3 Nordic interior layout

From the 1930s to the 1950s, Nordic furniture design was legendary development, the unique Nordic "functionalism" concept is also perfect during this period, and the emergence of a number of furniture designers, driven by these masters, Nordic countries of the furniture design gradually formed a relatively uniform style and swept the world. Today, Scandinavian designers and manufacturers are no longer focused solely on the appearance of furniture, but rather focus on presenting the designer's unique style. For a long time, the "Nordic Design", which is known as "concise, comfortable and modern", is indeed a style that can not be overlooked in the world design field. To understand this style in a comprehensive way, Style behind the reasons, because a regional design style is produced by the region's natural environment and social factors and other conditions. Now, the Nordic furniture design concept everywhere, its furniture brand modern and practical style quickly occupied the furniture market, what is the reason for the Nordic furniture in just half a century so deeply rooted it? This article will analyze the reasons for this.

\section{Analysis of the characteristics of Nordic furniture design}

Scandinavians have been struggling to learn to live in harmony with the surrounding environment. The grimy climate and limited raw materials have created the ability of the Nordic people to save their lives. The lack of natural resources has produced endless creativity and self-sufficiency. The They are constantly looking for inspiration from the rest of the world, but they also by virtue of their own feelings and local materials to be improved, and the superb appearance of technology and mass production together. In the 1930s, when functionalism began, due to geographical limitations, functionalism in the Nordic epidemic is unusually slow, which makes them have time to fully learn from other parts of the world to promote the experience and lessons of functionalism, they believe that the industrialization process will Depriving them of their cultural 
traditions and actively taking measures that include preserving crafts traditions and using indigenous materials. The Nordic forest coverage is extremely high, and the Nordic early life tools are made from birch. (Figure 4), while the traditional elements of furniture technology and materials along with the development of production in the region at the same time, and gradually led to the people of the region's existing design respected.

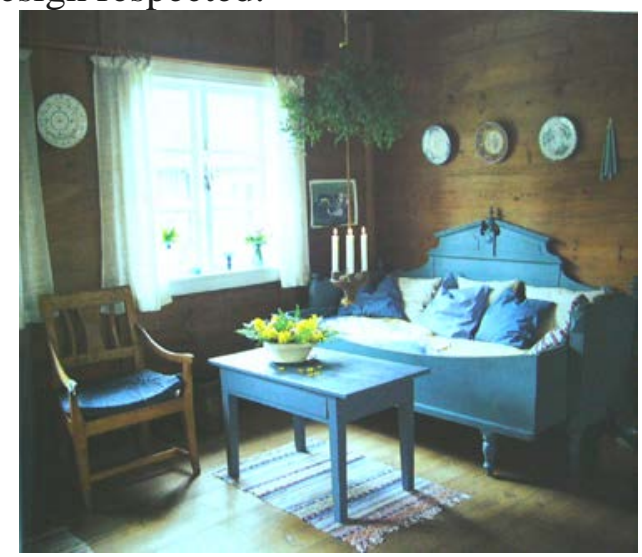

Figure 4 Nordic birch furniture

Prior to the beginning of this century, most of the Nordic furniture relied heavily on purchase orders by the beginning of the twentieth century, and only cheap and simple furniture was produced in volume. High consumption of the royal furniture is the most famous designers by the sample design to produce. The number of early Nordic furniture and consumer groups, determines the continuation of the traditional essence of the Nordic furniture. Craftsmen are crafted, designed to produce a small but exquisite furniture, presented to the royal family, this production method, although the promotion of furniture is extremely unrealistic, but for the traditional furniture manufacturing process heritage laid a solid foundation.

Nordic barren land and cold climate make their land lacking rich trees and resources, and in normal circumstances, such a situation can not meet the design requirements of diversification. But a single material is given a rich shape and function, more easy to form a local style.

The "Mushroom Series" seat designed by Elohano (Figure 5) shows the Nordic designer's attention to the local material. In 1954, Elono had a chance to learn to knit baskets and, by chance, find that inverted baskets could be used as a good seat, which was the inspiration for the birth of the famous "Mushroom" series of seats. The use of the rattan in the furniture design, is a breakthrough material, and "inverted basket" shape more of this breakthrough to increase the wonderful place. "Mushroom" chair of the birth and the advent of its series of products and the Nordic furniture in the world to show its local character of a sign.

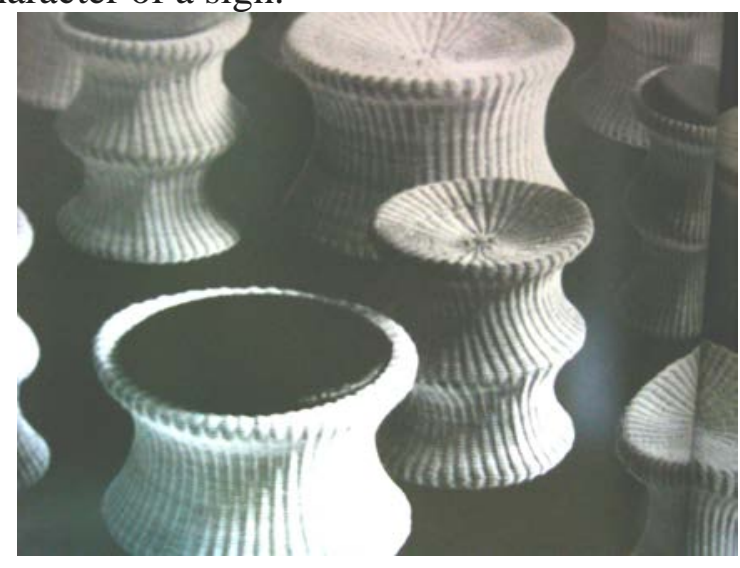

Figure 5 "Mushroom Series" seats

In 1951, Finnish designer Nomis Naimi designed the "horseshoe" sauna stool for the Finnish Palace Hotel. (Figure 6) The combination of plywood and teak, although deviated from the typical design of traditional French furniture, more performance of the early 1950s popular modernist strict 
rationalist principles. But Norris Nami's sauna stool quickly became a unique example of a unique integrated modernist and traditional. The simple design of this stool is closely linked to its use. It has a visible node can be directly, it is obvious that people think of folk art. Bathing as an important industry in Finland, and the placement and use of this sauna chair, both the performance of the natural purity of the Finnish furniture without modification of the original also showed the development of society and the degree of modernization.

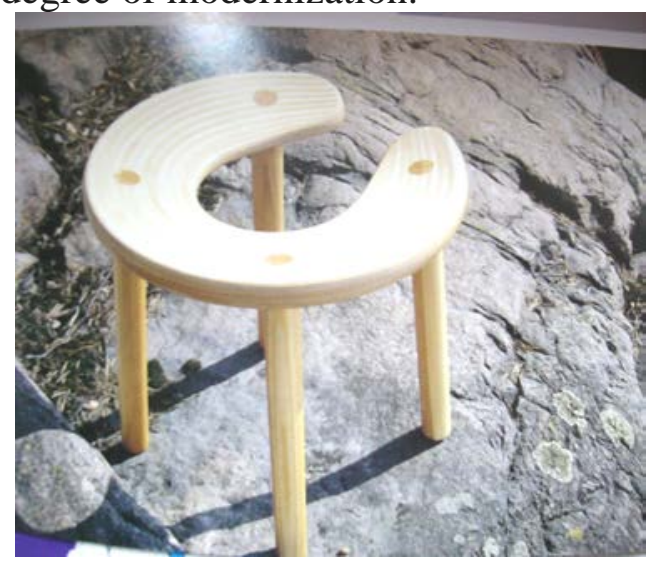

Figure 6 "horseshoe-shaped" sauna stool

Nordic furniture, from purely local to the designer's efforts and modernism after the convergence, all reflect the Nordic furniture design changes and more characteristic of the process. But this change is still local and small, Nordic furniture design can sweep the world and won the hearts of people have to return to the public and many designers and people's efforts.

Compared with ancient Egypt, China and other ancient civilizations, the Nordic are quite young. When a thousand years ago, China is experiencing a high degree of development of the Tang and Song dynasties, the Nordic is in the nomadic and hunting stage, daily utensils are basically from the forest and the lake directly to, or slightly processing, but each kind of supplies are Is the "functionalism" of the product. After the Second World War, the simplicity was praised as the most ideal form of the object. Decorative and intricate forms are called romanticism in the 1940s, and in the ensuing decades, people have taken a negative attitude towards both decorative and intricate forms. Simple and simple objects are also considered to have a clear functionality. This period of time it is expressed by the material integrity, authenticity and structure of the clarity.

The new multi-function chair (see Figure 7) is a representative of Nordic "functionalism". The design is the perfect interpretation and combination of the two mutually influential creative thinking of postwar postmodernism. The prototype of the chair was first called "double-legged chair" made of black leather chrome-plated steel pipe. This chair was later named "Milan Chair", which is a truly advanced design, simple form, smooth surface and detailed use of the material is "double chair" features.

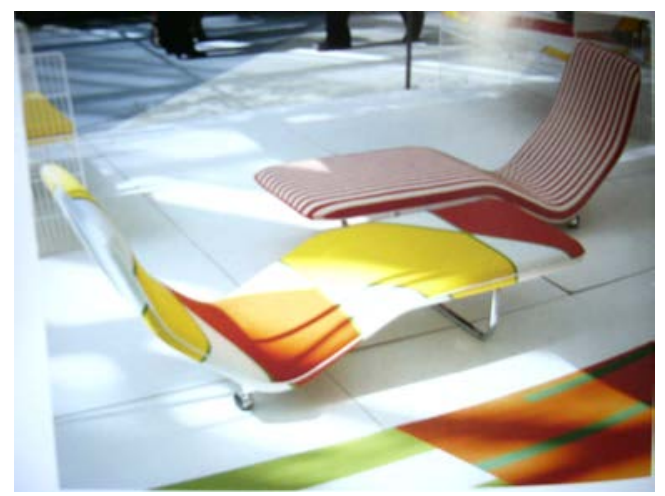

Figure 7 Multi-function chair

In the 1960s, two styles of Nordic furniture design were established: on the one hand, it was based on the continuation of traditional wooden furniture; on the other hand, furniture design 
following the international trend, like steel and plastic fibers, began to develop.

Nomis Nami's 001 is a model that follows the international trend. In the late 1960s, Nomis Naimi designed a long sofa, the form and material are the most simplified. The 001 chair consists of a seat part and a metal pipe at the foot, and all the extra parts are omitted, and the form of the seat just conforms to its function. The simple and soft seat is designed from an ergonomic point of view, and it is almost lying on the floor. This sofa is the culmination of its designers' reading of modernism. Seat part of the cable, simplified lines show "male masculinity", through the whole simple form, Changsha made a modern pursuit of high quality.

Aloeño in the 001 chair after the design of unique "soap chair" (Figure 8), which is full of creative and childlike seats, but also a bold exploration of the furniture materials and colors, advancing the Nordic The development of furniture design style. "Soap chair", also known as "baby chair", it can be viewed from any angle, and has a sense of beauty. In the basic composition of the product, "soap chair" than the minimalism even more concise, than the Nordic counterparts designer window works more concise. This legless seat is made of glass-reinforced polyester fiber, only for two parts of the forging, it has the characteristics of modern art, but also humor to show the shape of candy or bubble gum, in addition to bright colors are more Add a modern effect. "Soap chair" is a modern rocking chair deformation, from the appearance point of view, like a piece of thumb on the printed candy. "Soap chair" is not only a designer of the Nordic modern furniture, an important innovation, but also relative to the whole world.

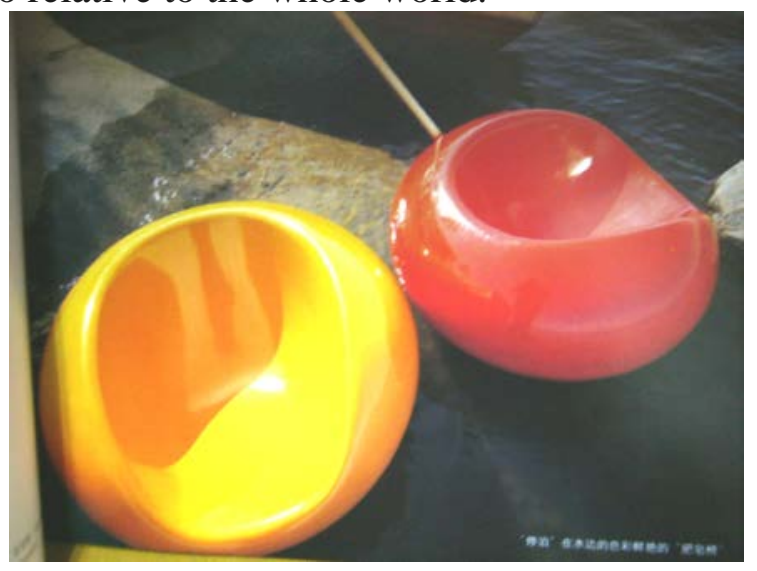

Figure 8 Soap chair

When furniture manufacturing is increasingly tending to industrial scale production, designers feel that manufacturers are only focused on profit margins and mass production, while manufacturers feel that designers are only interested in designing high-end furniture. In the late twentieth century, a small, elegant table and chair series made from beech plywood from Scandinavia achieved large-scale production, practical and high quality, and the price was relatively cheap. This was the balance between high quality furniture and civilians. But also the new era of "new" and "old" combination of points.

The most interesting feature of the development of furniture design is mainly to go their own way, not by the vagaries of the various trends, Nordic furniture design is not blindly catch up with the trend, not whimsical control, they continue to develop and improve Nordic modernist style, attention to the production process and quality, in-depth analysis of human structure, aesthetics, use and needs.

Nordic design emphasizes people-oriented, emphasizing practical, simple and coordinated with the traditional style. Design to create culture, design also represents a new civilization. Technology and human nature go hand in hand with the Nordic design will be more abundant human daily life.

\section{References}

[1] Pamela Diaconis. Scandinavian pastoral scenery. Tianjin Science and Technology Translation Publishing Company published. 
[2] John Pyle. World interior design history. China Construction Industry Press.

[3] Sven. Tiberi. Swedish residential research and design. China Construction Industry Press.

[4] "colorful" magazine planning. The so-called Nordic style, 2002,6.

[5] Fang Hai. Luo Ping Jia. Nordic modern design of the banner - Angi. Nori Si meters. China Construction Industry Press .2004

[6] Fang Hai. Luo Ping Jia. Nordic romantic master of design - Ai Luo. Anio. China Construction Industry Press .2004

[7] Fang Hai. Finnish modern furniture. China Construction Industry Press .2004

[9] Nordic Modern Design Series. Danish Biography - Furniture and Interior Design. Heilongjiang Science and Technology Press. 